\title{
Measurement of $(n, \alpha)$ cross section for set of structural material isotopes
}

\author{
Vitaly Khryachkova, Alexander Gurbich, Tatiana Khromyleva, Ivan Bondarenko, Vladimir Ketlerov, and Pavel \\ Prusachenko
}

Institute for Physics and Power Engineering (IPPE), Obninsk, Russia

\begin{abstract}
A novel spectrometer was developed and used to measure the cross section for the $(\mathrm{n}, \alpha)$ reaction at IPPE. Direct measurements of the $\alpha$-particles yield from solid isotopic pure targets of 50, 52 and 53 chromium, 54 and 57 iron, 60 nickel, and 64 zinc were carried out in the neutron energy range from 4.7 to $7.2 \mathrm{MeV}$. For some isotopes the $(\mathrm{n}, \alpha)$ reaction cross-section for neutron energies less than $14 \mathrm{MeV}$ were measured for the first time. The result of the comparison of new experimental data with the evaluated data from libraries ENDF/B VII, JENDL 4.0, JEFF 3.1, ROSFOND 2010 and BROND 3 and with the experimental data of other authors is presented.
\end{abstract}

\section{Introduction}

In spite of the fact that structural materials are widely used in nuclear power engineering, the data for $(\mathrm{n}, \alpha)$ reaction cross section given in different libraries are significantly discrepant. Therefore, new experimental efforts are necessary to resolve the problem. Unfortunately the $(\mathrm{n}, \alpha)$ reaction for many structural material elements produce non-radioactive residual nuclei in output channel and so the classical activation technique cannot be used. A novel spectrometer was developed and used to measure the cross section for the $(\mathrm{n}, \alpha)$ reaction at IPPE.

\section{Experimental set up}

The targets were loaded into an ionization chamber equipped with a Frisch grid (see Fig. 1). Ionization chamber was filled with $97 \% \mathrm{Kr}+3 \% \mathrm{CH}_{4}$ or $97 \% \mathrm{Kr}$ $+3 \% \mathrm{CO}_{2}$ at a pressure of 3 at. Both mixtures produce a lot of background particles under fast neutron irradiation. For methane they are recoil protons, for carbon dioxide they are $\alpha$-particles from ${ }^{16} \mathrm{O}(\mathrm{n}, \alpha)$ reaction. Depending on Q-value of an investigated reaction more suitable gas mixture was chosen. The use of digital methods of signal processing makes it possible to select useful signals from a great number of background pulses.

Our previous experience in mounting the target on the chamber's cathode showed that during its irradiation by fast neutrons, the cathode material became an intensive source of $\alpha$-particles appearing as a result of $(\mathrm{n}, \alpha)$ reactions occurring on its material components and the oxygen and nitrogen dissolved on its surface.

To minimize the background, we used the experience of investigations of $(n, \alpha)$ reactions on the components of the ionization chamber's working gas $[1,2]$. The target was mounted on gold filaments fastened to the chamber's first guard electrode. As a result, the target was placed within

a e-mail: hva@ippe.ru the cathode-grid space at a distance of $1 \mathrm{~cm}$ from the cathode (see Fig. 1). The gold filaments served not only to fix the target, but also to preserve the electric potential corresponding to the position of the target.

Under these conditions, signals from the $\alpha$-particles generated on the target's surface can be distinguished from signals of particles occurring on the cathode or in the operating gas itself. For that, we have to measure the drift time for the electrons most distant from an anode. If $\alpha$-particle is generated on the cathode, the last electrons come from the area near the cathode; i.e., they overcome a distance of $4 \mathrm{~cm}$. When the particle starts from the target, the electrons must cover only the three centimeters separating the target and the anode. Analysis of the drift time allows us to separate particles by their places of origin, and consequently to reduce the background of parasite reactions. Such a detailed analysis of the shape of the anode and cathode signals can, however, only be performed by digital signal processing.

Signals from the anode and cathode of the ionization chamber were supplied after amplification to LeCroy 2262 pulse-shape digitizer that transformed the input signal into a sequence of numbers corresponding to the amplitudes of the input signal at different instants of time (see Fig. 2). The digitized signals were stored on the hard disk of a personal computer for subsequent processing.

The measurements described in this work were performed on the EG-1 accelerator of IPPE. Neutrons were generated in the $\mathrm{D}(\mathrm{d}, \mathrm{n})$ reaction on a solid deuterated titanium target with a thickness of $1 \mathrm{mg} / \mathrm{cm}^{2}$. We used a parallel plate ionization chamber containing a thin ${ }^{238} \mathrm{U}$ layer to monitor the neutron flux. The uranium target was mounted on the common cathode of the two chambers in back-to-back geometry with the main chamber (see Fig. 1). The ${ }^{238} \mathrm{U}$ isotope content in the target was $99.99 \%$. The ${ }^{238} \mathrm{U}$ mass of $4.60 \mathrm{mg}$ was determined by $\alpha$-spectrometry. The dead time in the monitor and main registration channels were identical due to certain features of our

(C) The Authors, published by EDP Sciences. This is an Open Access article distributed under the terms of the Creative Commons Attribution License 4.0 (http://creativecommons.org/licenses/by/4.0/). 


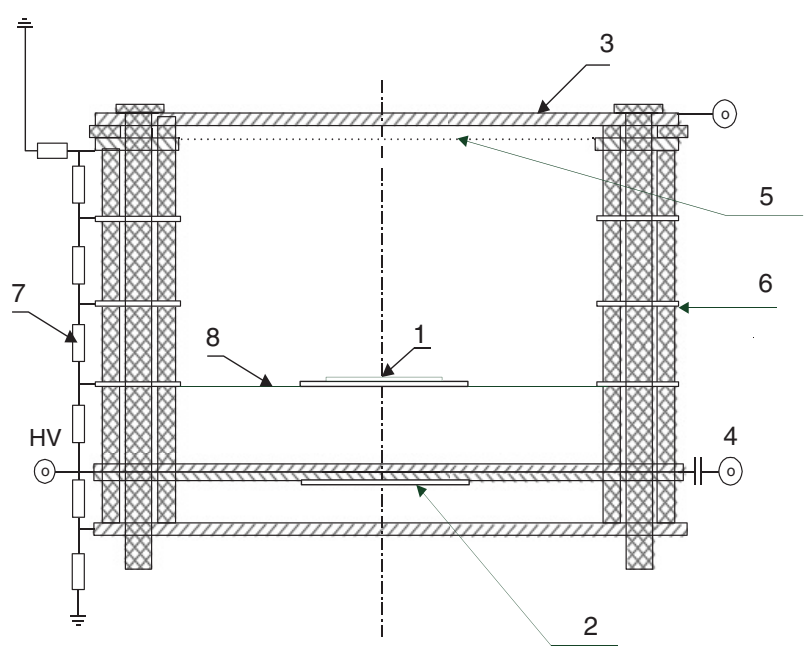

Figure 1. Detector construction scheme. (1) Target; (2) ${ }^{238} \mathrm{U}$ target; (3) PIC (Pulse Ionization Chamber) anode; (4) common cathode; (5) Frisch grid; (6) guard electrodes; (7) divider; and (8) gold filaments.

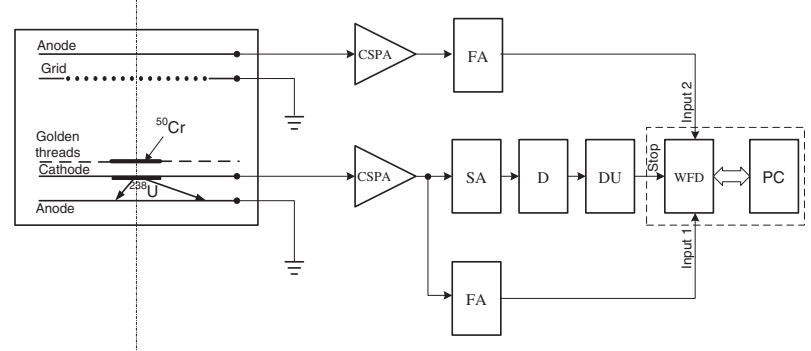

Figure 2. Block diagram of the detector and electronics. CSPA - charge sensitive preamplifier; SA - spectrometric amplifier; D - discriminator; DU - delay unit; WFD - waveforms digitizer; FA - fast amplifier.

method for collecting information. The neutron flux was measured in an area very close to the location of the target. Correction for the difference between the uranium and target's positions $(1 \mathrm{~cm})$ was carried out at the processing stage.

\section{Data analyses}

Our software enables us to extract the following information from the digital signals: the amplitudes of the anode and cathode signals, and times of their beginning and end. Combined analysis of the information obtained for each event provides us with information on the energy of a particle, its place of origin, and its type. Every of the measured parameters allow us to reduce considerably the background contribution and, as consequence, to increase the reliability of determination of the number of events belonging to the investigated reaction.

This way of signal acquisition and processing allows us to determine a number of parameters of the recorded event at once. During the processing the signal amplitude, the drift of electrons to the anode, the length of the projection of the particle track on the axis of the chamber were determined. Figure 3 shows the two-dimensional spectrum, where the $\mathrm{X}$-axis is an amplitude of the anode signal, the Y-axis is the electron drift time. From the figure, it is clear that all

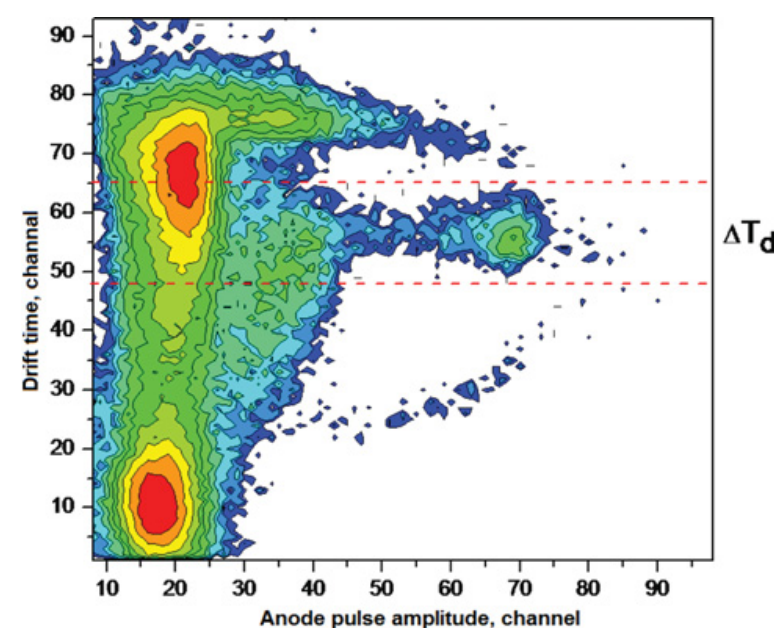

Figure 3. A two-dimensional spectrum, where the $\mathrm{X}$-axis is the amplitude of the anode signal, the Y-axis is the electrons drift time obtained after background suppression.

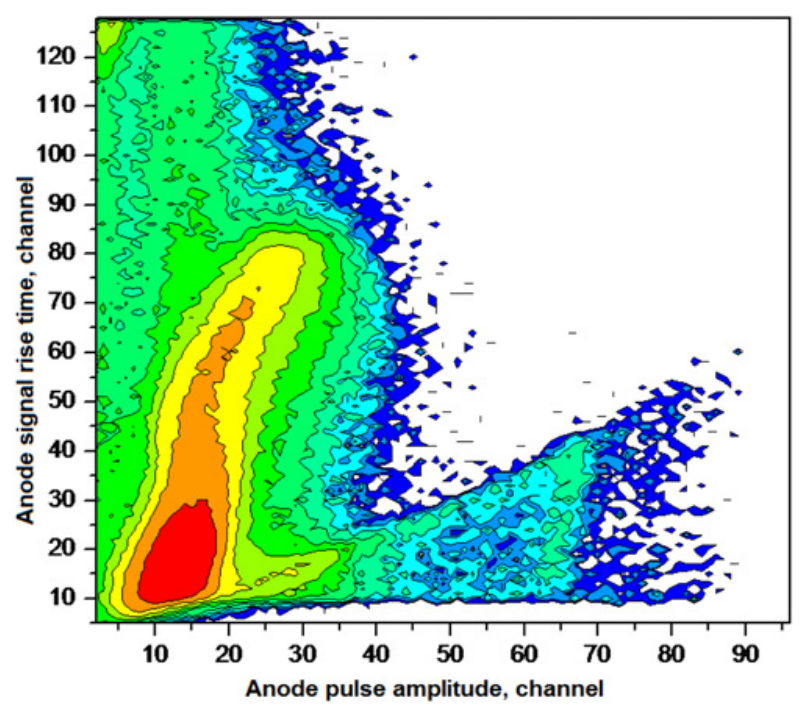

Figure 4. A two-dimensional spectrum, where the $\mathrm{X}$-axis is the amplitude of the anode signal, the Y-axis is the rise time of the signal obtained for ${ }^{50} \mathrm{Cr}$ target (neutron energy of $6.5 \mathrm{MeV}$ ).

of the events are divided into 3 groups - the events that took place at the cathode (upper part of the spectrum), the events that took place in the studied target (the middle part of the spectrum), and the events happened in the working gas (the lower part of the spectrum).

By choosing the window for "electron drift time" parameter we can select events originated from the target (shown on the Fig. 3 by the dashed line). This method allows us to suppress significantly the contribution of background reactions, occurring on the structural elements of the chamber and in its working gas. Figure 4 shows the two-dimensional spectrum, where the $\mathrm{X}$-axis is the amplitude of the anode signal and the Y-axis is the rise time of the anode signal. The rise time parameter of the anode signal allows us to separate particles of different types. Indeed the rise time parameter of the anode signal is directly connected with the particles path projection on the symmetry axis of the chamber. At the same amplitude of the anode signal (particle energy), the lighter particles will have longer range. 


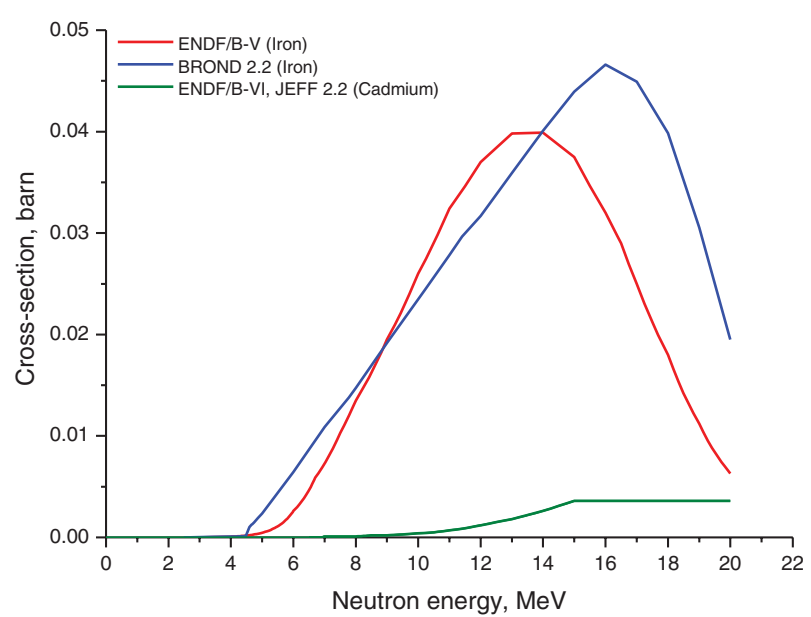

Figure 5. Theoretical estimates of the cross sections of $(n, \alpha)$ reaction on natural iron and cadmium.

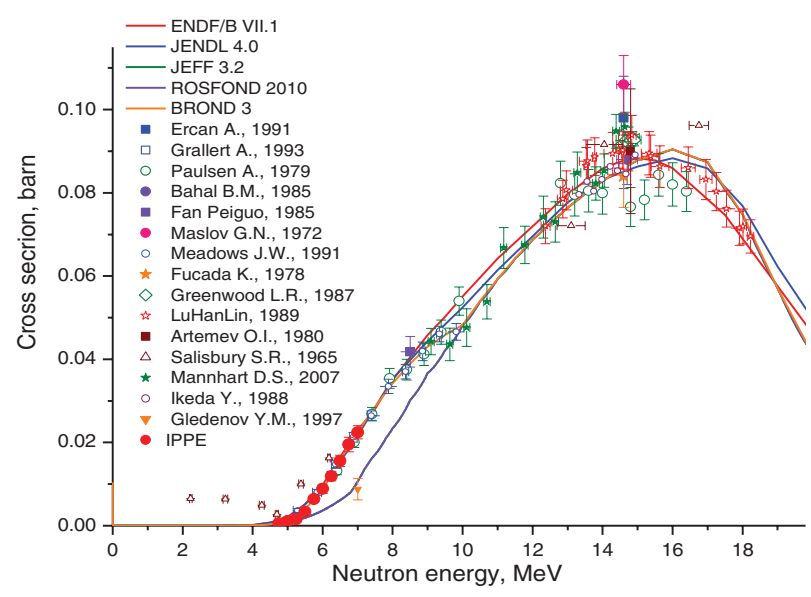

Figure 6. The results of measuring the cross section of ${ }^{54} \mathrm{Fe}(\mathrm{n}, \alpha){ }^{51} \mathrm{Cr}$ in comparison with the evaluated data ENDF/B VII.1, JENDL 4.0, JEFF 3.2, ROSFOND 2010 and BROND 3 libraries and the experimental data of other authors.

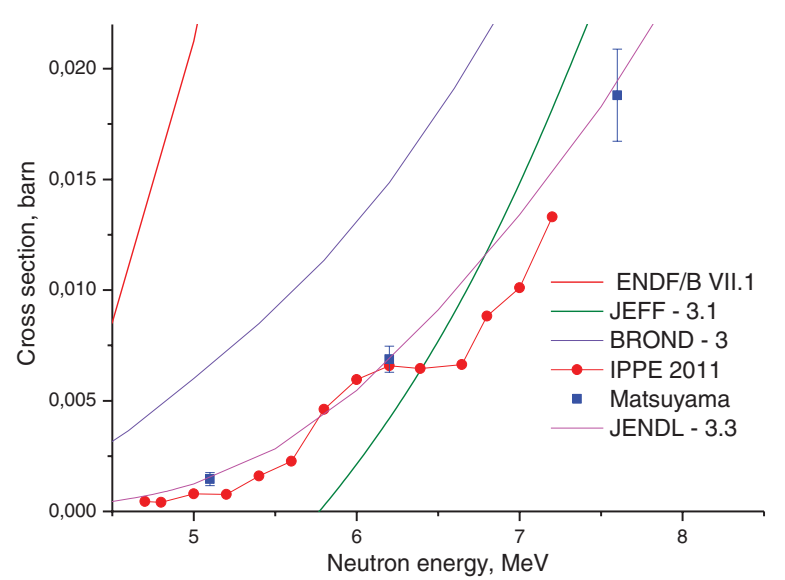

Figure 7. The cross section of the reaction ${ }^{50} \mathrm{Cr}(\mathrm{n}, \alpha)^{47} \mathrm{Ti}$ compared with the evaluated data libraries ENDF/B VII.1, JENDL 3.3, JEFF 3.1 and BROND 3 and the experimental data of other authors.

In Fig. 4 a group of particles with short range ( $\alpha$-particles) and with long range (protons and electrons)

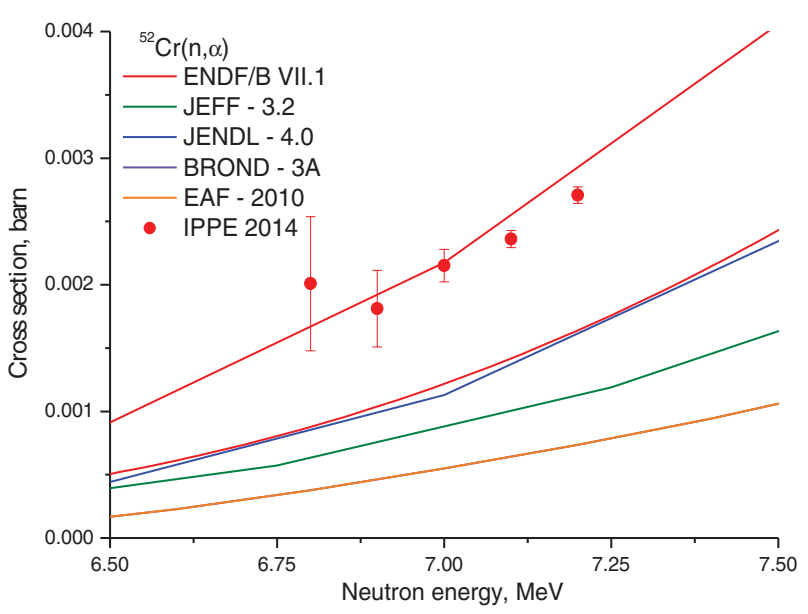

Figure 8. The results of the measured cross section of the ${ }^{52} \mathrm{Cr}(\mathrm{n}, \alpha)^{49} \mathrm{Ti}$ compared with evaluated data libraries ENDF/B VII.1, JENDL 4.0, JEFF 3.2 and BROND 3.

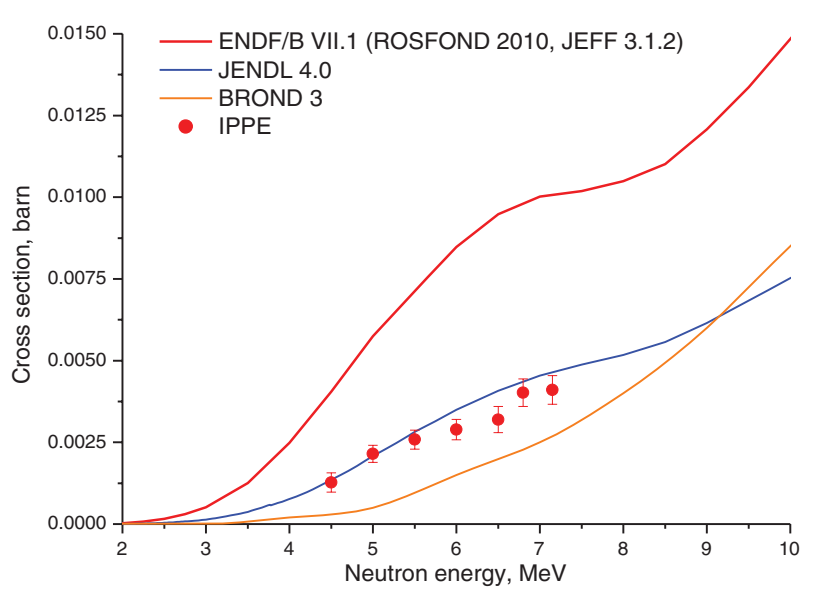

Figure 9. The results of the measured cross section of the ${ }^{53} \mathrm{Cr}(\mathrm{n}, \alpha)^{50} \mathrm{Ti}$ compared with evaluated data libraries ENDF/B VII.1, JENDL 4.0 and BROND 3.

can be seen. For $\alpha$-particles (due to their short range in the gas) there is a small value of the "rise time of the anode signal" parameter. Cutting particles with large values of the rise time of the anode signal we discriminate events caused by the particles other than $\alpha$-particles. This way of selection of events allows us to reduce the background and to distinguish the events corresponding to the investigated reaction.

Traditionally, the electrodes of the ionization chamber are made of stainless steel. This material is corrosion resistant and it allows achieving a good vacuum in the chamber. However, to study the $(\mathrm{n}, \alpha)$ reaction for iron, chromium and nickel isotopes it is obviously not suitable as it contains incomparably greater amount of studied nuclei than there are in a thin spectrometric target. To solve this problem cadmium covers of the sides of the electrodes which faced the sensitive volume of the chamber were used. The excitation functions of $\alpha$-production for natural iron and cadmium are shown in Fig. 5. The figure shows that the use of cadmium can significantly reduce the background caused by electrodes in the whole energy range of neutrons we are interested in. 


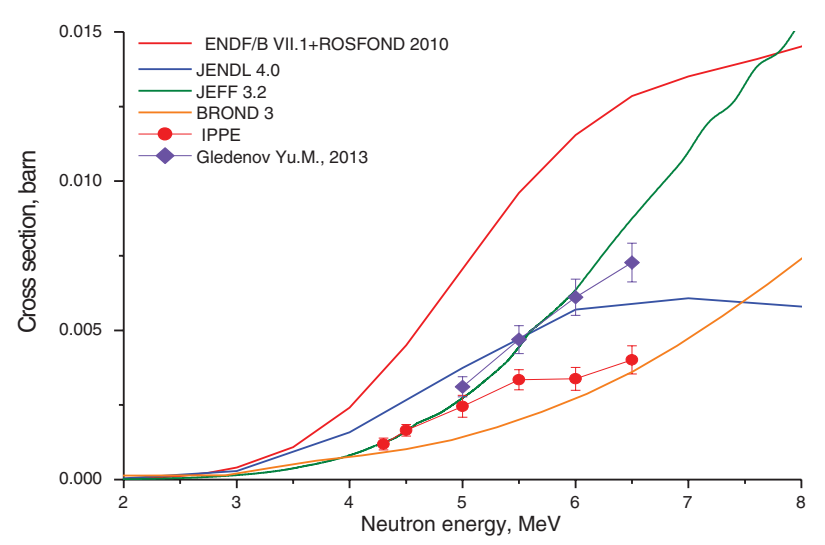

Figure 10. The results of the measured cross section of the ${ }^{57} \mathrm{Fe}(\mathrm{n}, \alpha){ }^{54} \mathrm{Cr}$ compared with evaluated data libraries ENDF/B VII.1, JENDL 4.0, JEFF 3.2, ROSFOND 2010 and BROND 3.

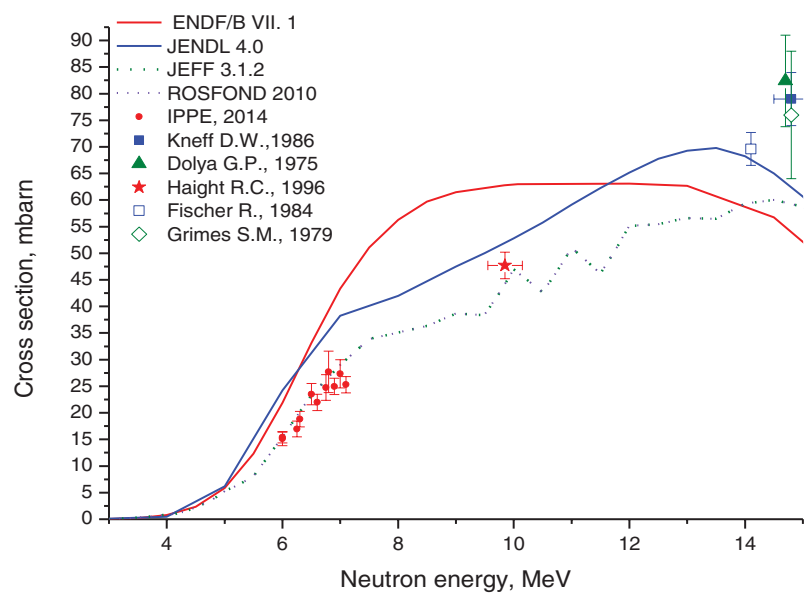

Figure 11. The results of the measured cross section of the ${ }^{60} \mathrm{Ni}(\mathrm{n}, \alpha){ }^{57} \mathrm{Fe}$ compared with evaluated data libraries ENDF/B VII.1, JENDL 4.0, JEFF 3.1.2 and ROSFOND 2010.

\section{Results}

The developed method was used to measure the cross section of the ${ }^{54} \mathrm{Fe}(\mathrm{n}, \alpha){ }^{51} \mathrm{Cr}$ reaction. The results are shown in Fig. 6. For this reaction, there are a number of experiments carried out using classical activation method and their results are in a good agreement with each other. This allows us to use this reaction as a reference to confirm the correctness of the procedures of the determination of the number of nuclei in the target (which was made by Rutherford backscattering spectrometry) and the number of registered events.

As follows from the Fig. 6, our data coincide with the data of other authors that allows us to make a conclusion about the correctness of the entire process of the crosssection determination of the studied reaction.

Detailed studies of the excitation function of the reaction ${ }^{50} \mathrm{Cr}(\mathrm{n}, \alpha){ }^{47} \mathrm{Ti}$ in the neutron energy range from 4.5

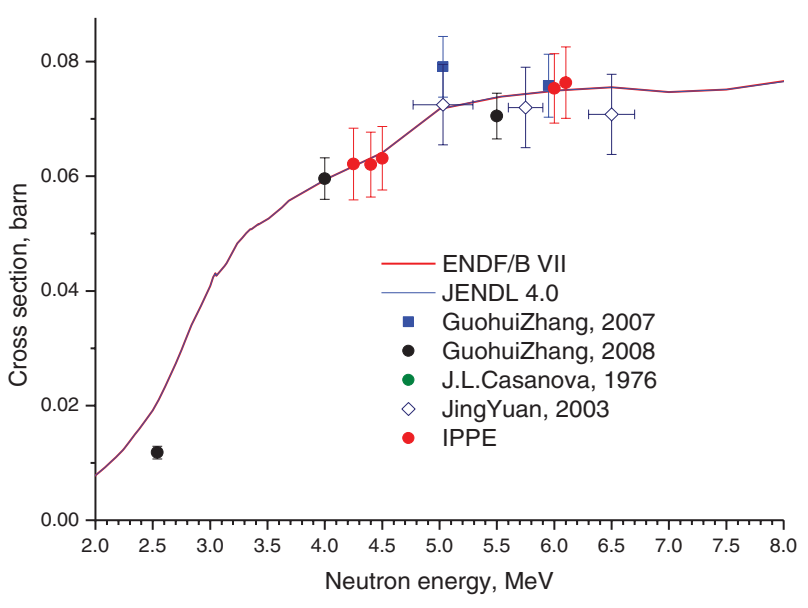

Figure 12. The results of the measured cross section of the ${ }^{64} \mathrm{Zn}(\mathrm{n}, \alpha){ }^{61} \mathrm{Ni}$ compared with evaluated data libraries ENDF/B VII.1, JENDL 4.0.

to $7.2 \mathrm{MeV}$ were performed with the use of the developed spectrometer. The results are shown in Fig. 7. It should be noted that the results are in a good agreement with the data given in [3]. For the evaluated data given in the different libraries there is a huge discrepancy. Our data are in a satisfactory agreement with estimates given by the library JENDL 4.0 and BROND 3, and disagree significantly with the ENDF / B VII.1 evaluation.

Figure 8 shows the results of the measurements of the cross section for the reaction ${ }^{52} \mathrm{Cr}(\mathrm{n}, \alpha)^{49} \mathrm{Ti}$. There is no other experimental data for this energy region. The closest cross sections were predicted by ENDF/B VII.1 library. Data of other libraries are significantly lower than experimentally observed values.

The results of the cross section measurements for ${ }^{53} \mathrm{Cr}(\mathrm{n}, \alpha){ }^{50} \mathrm{Ti}, \quad{ }^{57} \mathrm{Fe}(\mathrm{n}, \alpha){ }^{54} \mathrm{Cr}, \quad{ }^{60} \mathrm{Ni}(\mathrm{n}, \alpha){ }^{57} \mathrm{Fe}$ and ${ }^{64} \mathrm{Zn}(\mathrm{n}, \alpha){ }^{61} \mathrm{Ni}$ reactions and the evaluated data given by the various libraries are shown in Figs. 9, 10, 11 and 12, respectively.

\section{Conclusion}

New digital technique for direct measurements of $\alpha$ particles originated from $(n, \alpha)$ reactions on a solid target was developed. New data for the ${ }^{50} \mathrm{Cr}(\mathrm{n}, \alpha)^{47} \mathrm{Ti}$, ${ }^{52} \mathrm{Cr}(\mathrm{n}, \alpha){ }^{49} \mathrm{Ti},{ }^{53} \mathrm{Cr}(\mathrm{n}, \alpha){ }^{50} \mathrm{Ti},{ }^{57} \mathrm{Fe}(\mathrm{n}, \alpha){ }^{54} \mathrm{Cr},{ }^{60} \mathrm{Ni}(\mathrm{n}, \alpha){ }^{57} \mathrm{Fe}$ and ${ }^{64} \mathrm{Zn}(\mathrm{n}, \alpha){ }^{61} \mathrm{Ni}$ cross-sections were obtained.

\section{References}

[1] G. Giorginis, V. Khryachkov et al. In Proc. of Int. Conf. NDST 2007 conference. Nice, 525 (2007)

[2] V. Khryachkov, I. Bondarenko et al. EPJ Web of Conferences. 21 (2012)

[3] M. Baba, N. Ito, I. Matsuyama et al. In Proc. of ND1994, Gatlinburg, 941 (1994) 